Supporting Information for:

\title{
Charge-Transfer Character in Excimers of Perylenediimides Self- Assembled on Anodic Aluminum Oxide Membrane Walls
}

\author{
Michele S. Myong, Jiawang Zhou, Ryan M. Young and Michael R. Wasielewski* \\ Department of Chemistry and Institute for Sustainability and Energy at Northwestern, \\ Northwestern University, Evanston, IL 60208-3113
}

\section{SYNTHESIS AND CHARACTERIZATION}

${ }^{1} \mathrm{H}$ nuclear magnetic resonance spectra were collected on a Bruker Avance III $500 \mathrm{MHz}$ system and chemical shifts are recorded in ppm $(\delta)$ and samples dissolved in deuterated chloroform. The matrix assisted laser desorption ionization - time of flight (MALDI-ToF) spectra were taken on a Bruker AutoFlex-III.

${ }^{1}$ H NMR Spectros copy.

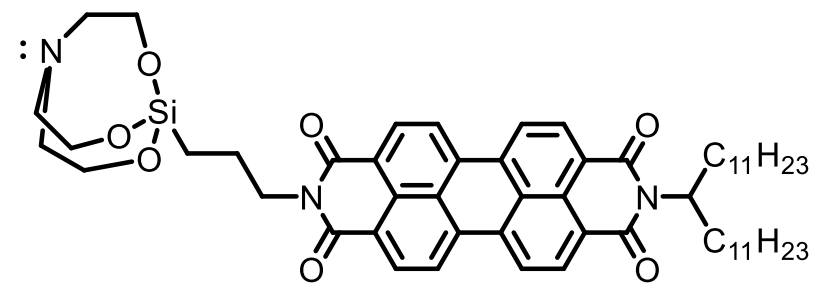

$N$-(12-tricosanyl)perylene-3,4:9,10-tetracarboxy-3,4-[(3-silatranylpropyl)amide]-9,10-imide

N-(12-tricosanyl)perylene-3,4:9,10-tetracarboxy-3,4-anhydride-9,10-imide $(0.44 \mathrm{~g}, 0.621 \mathrm{mmol})$ and (3-silatranylpropyl)amide ${ }^{1}(0.433 \mathrm{~g}, 1.86 \mathrm{mmol})$ were dissolved in pyridine and stirred for 1 hour. The solvent was removed by rotary evaporation and the resulting solid dissolved in dichloromethane and purified on a silica gel column using $\mathrm{CHCl}_{3}$ to elute the residual N,N'bis(12-tricosanyl)perylene-3,4:9,10-bis(dicarboximide). A mixture of $\mathrm{CHCl}_{3}$ : acetic acid (9:1) 
was used to the elute the product. The product was further purified using ${ }^{1} \mathrm{H} \mathrm{NMR}\left(\delta\right.$ in $\mathrm{CDCl}_{3}$, $500 \mathrm{MHz}): 8.63(\mathrm{~m}, 8 \mathrm{H}), 5.18(\mathrm{~m}, 1 \mathrm{H}), 4.21(\mathrm{t}, 2 \mathrm{H}), 3.74\left(\mathrm{t}, 6 \mathrm{H},-\mathrm{CH}_{2}-\mathrm{O}-\right), 2.78\left(\mathrm{t}, 6 \mathrm{H},-\mathrm{CH}_{2}-\right.$ N), $2.25(\mathrm{~m}, 2 \mathrm{H}), 1.86(\mathrm{~m}, 2 \mathrm{H}), 1.47(\mathrm{~m}, 2 \mathrm{H}), 1.12-1.33(\mathrm{~m}, 36 \mathrm{H}), 0.84(\mathrm{t}, 6 \mathrm{H}), 0.57(\mathrm{~m}, 2 \mathrm{H})$. HR-MS (ESI)(m/z): [M+] calc. for $\mathrm{C}_{56} \mathrm{H}_{73} \mathrm{~N}_{3} \mathrm{O}_{7} \mathrm{Si}$, 928.30; found, 928.5296.

\section{Fourier Transform Infrared Spectroscopy characterization of octadecyltriethoxysilane chains on the AAO surface.}
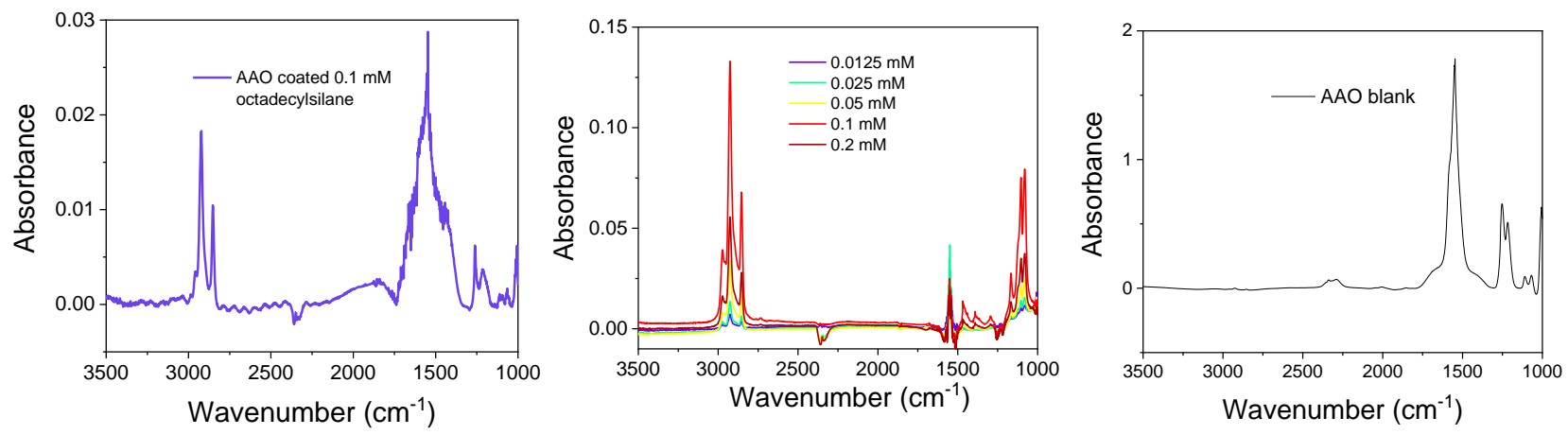

Figure S1. Left: FTIR spectrum of AAO coated in $0.1 \mathrm{mM}$ of octadecyltriethoxysilane for $24 \mathrm{~h}$. Middle: Concentration study of octadecyltriethoxysilane in carbon tetrachloride $\left(\mathrm{CCl}_{4}\right)$. Right: FTIR spectrum of uncoated AAO which exhibits no stretch at $\sim 3000 \mathrm{~cm}^{-1}$. 


\section{Scanning Electron Microscopy Images of Anodic Aluminum Oxide Membrane}
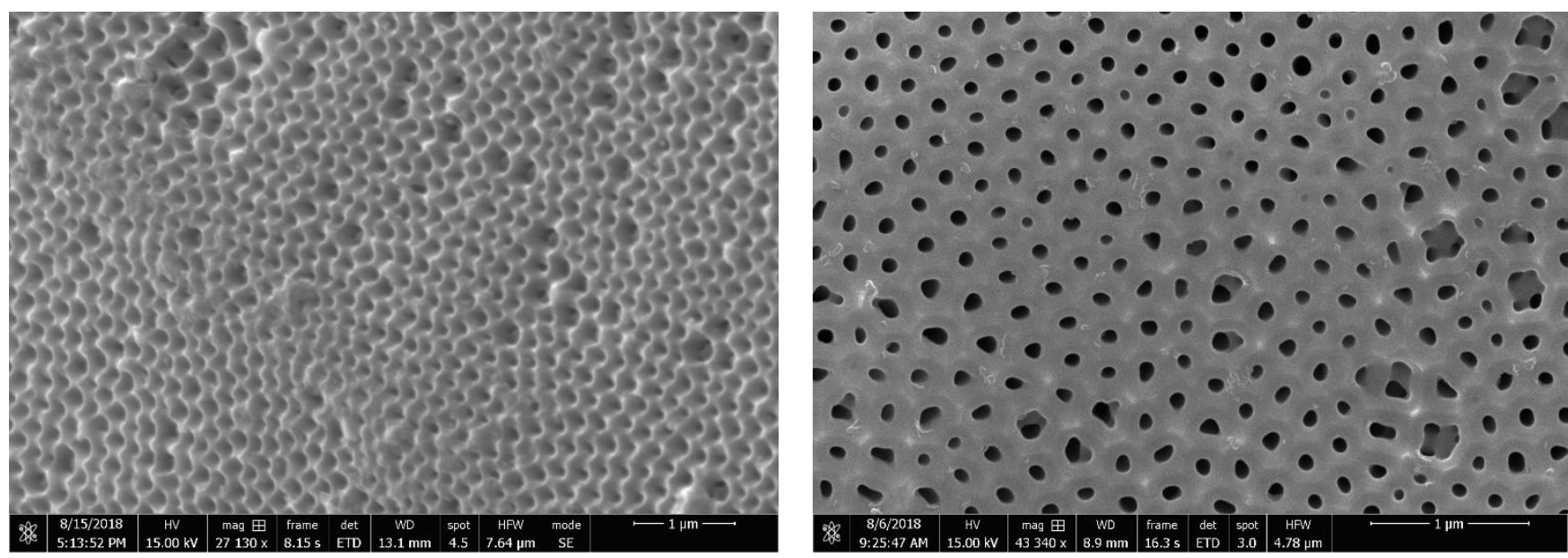

Figure S2. Left: Scanning electron microscope (SEM) image of the edge of an AAO membrane. Right: SEM image of the top of an AAO membrane.

\section{Additional Steady-State Absorption Spectroscopy}

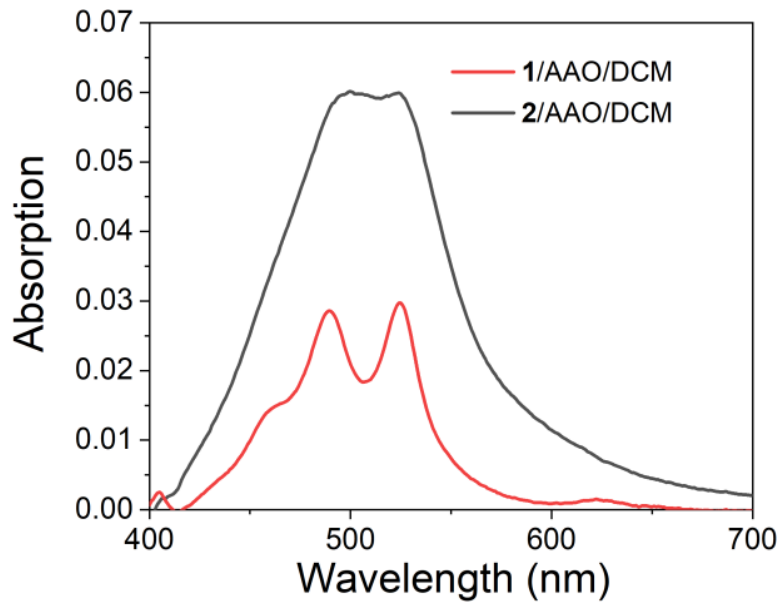

Figure S4. Unnormalized UV-Vis spectra of 1/AAO/DCM and 2/AAO/DCM. 


\section{Additional Transient Absorption Spectroscopy}
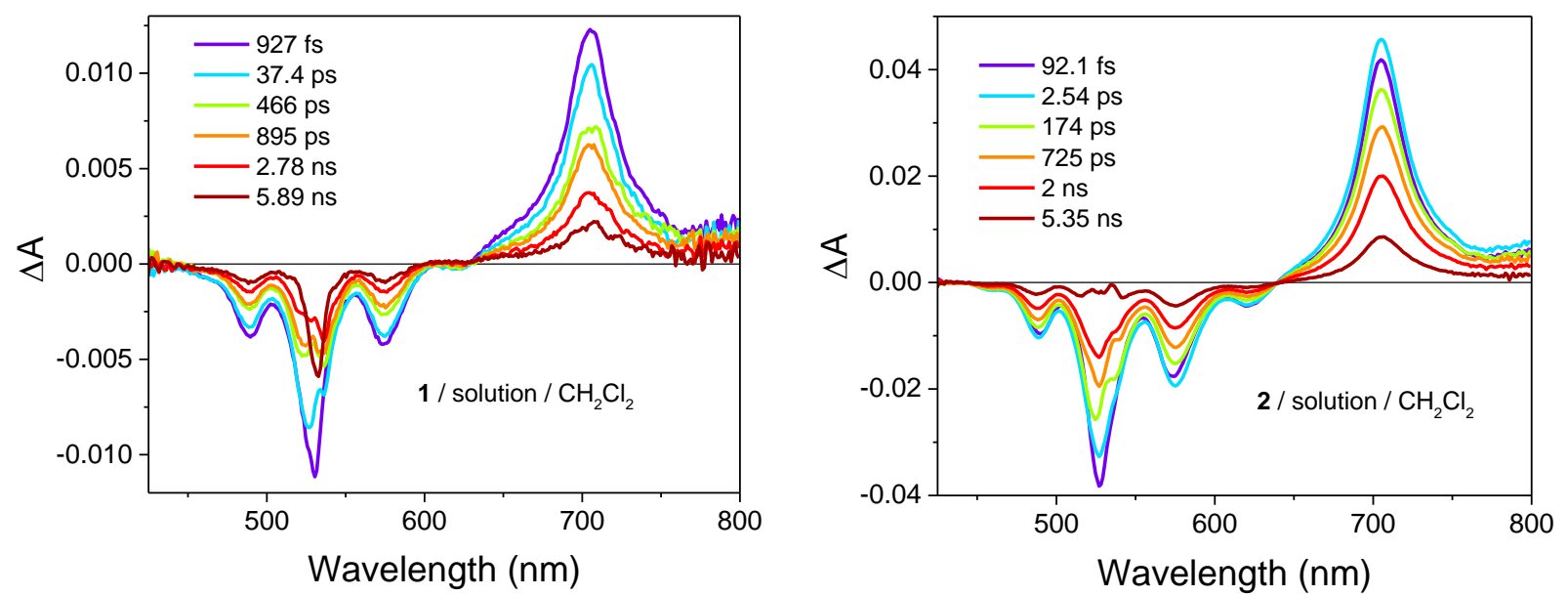

Figure S3. TA spectra for a solution of 1 (left) and 2 (right) in DCM acquired with $\lambda_{e x}=534 \mathrm{~nm}, \sim 110 \mathrm{fs}$ pulses.
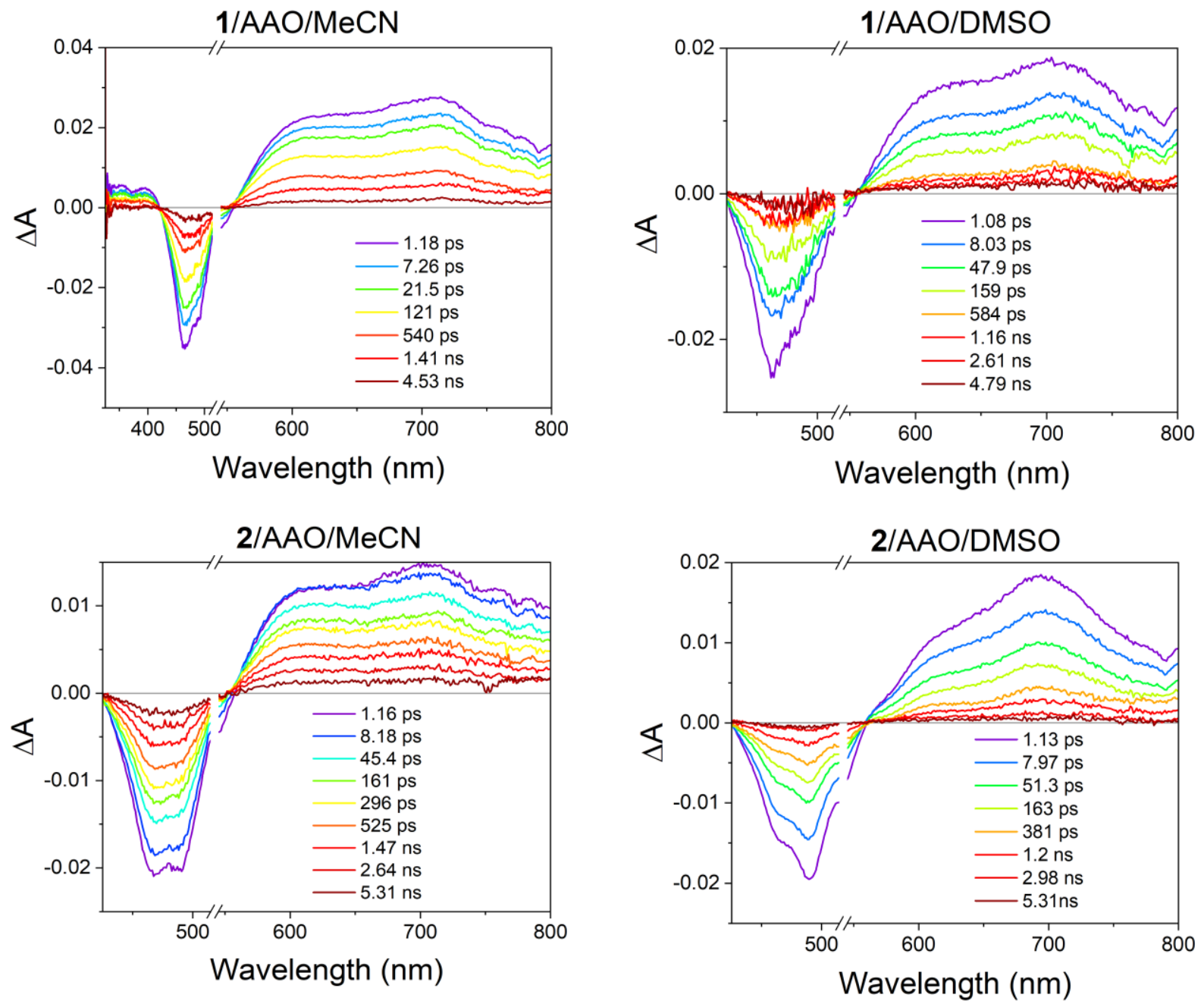

Figure S4. TA spectra for 1/AAO and 2/AAO in MeCN and DMSO. 

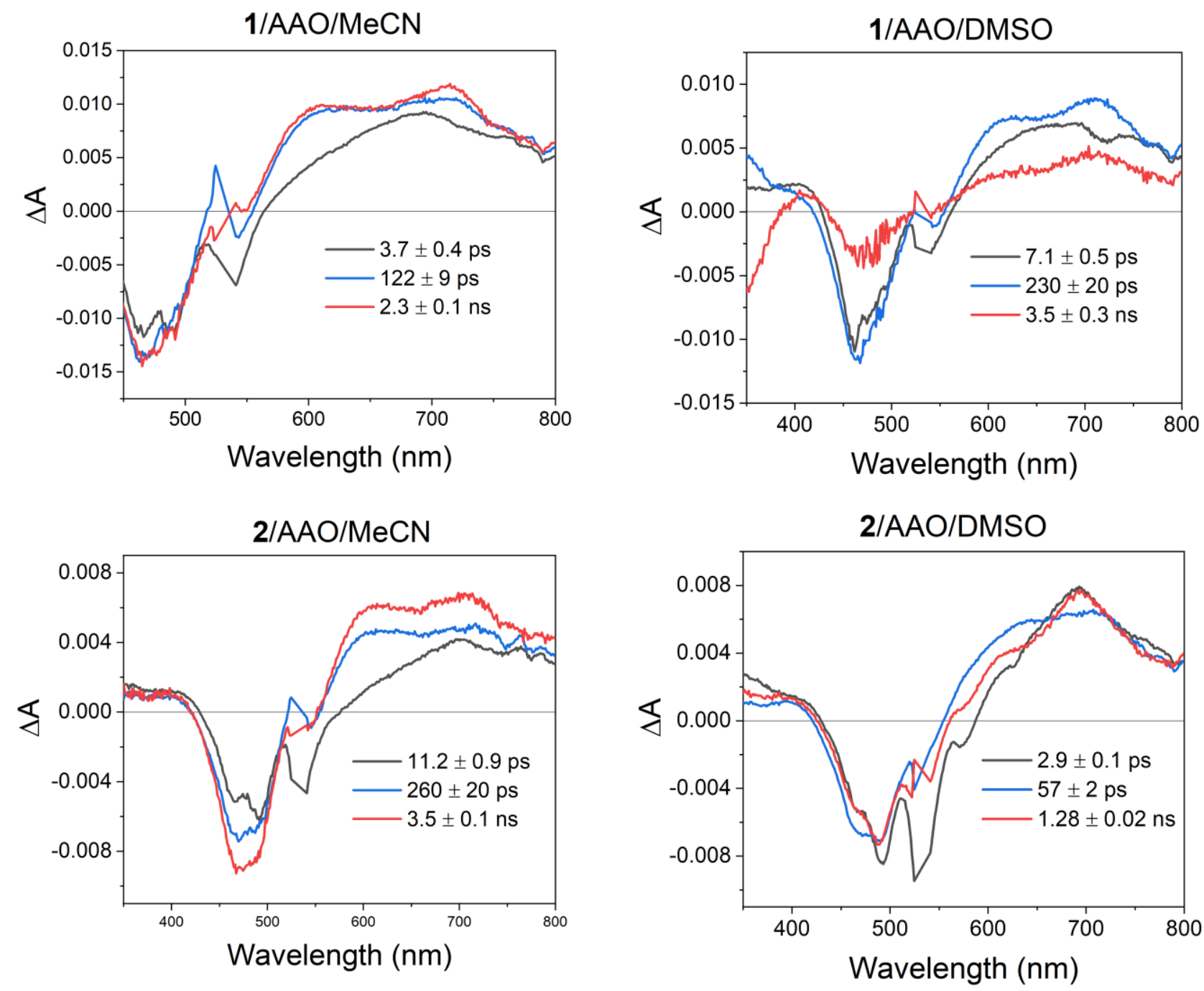

Figure S5. Decay-as sociated spectra for 1/AAO and 2/AAO in MeCN and DMSO.

Kinetic Analysis of fsTA Data: Prior to kinetic analysis, the fsTA data were background/scattersubtracted and chirp-corrected, and the visible and NIR data sets are spectrally merged (Surface Xplorer 4, Ultrafast Systems, LLC).

The kinetic analysis was performed using home written programs in MATLAB and was based on a global fit to selected single-wavelength kinetics. The time-resolution is given as $w=300$ fs (full width at half maximum, FWHM); the assumption of a uniform instrument response across the frequency domain and a fixed time-zero $\left(t_{0}\right)$ are implicit in global analysis. Each wavelength 
was given an initial amplitude that is representative of the spectral intensity at time $t_{0}$ and varied independently to fit the data. The time/rate constants and $t_{0}$ are shared between the various kinetic data and are varied globally across the kinetic data in order to fit the model. We globally fit the dataset to a set of three exponential decays and use the resultant populations to deconvolute the dataset and reconstruct decay-associated spectra. The MATLAB program convolutes the solutions with a Gaussian instrument response function with width $w$, before employing a least-squares fitting using a Levenberg-Marquardt or Simplex method to find the parameters which result in matches to the kinetic data. Each function corresponds to a given population with a well-defined temporal evolution. The raw data matrix is deconvoluted with the resultant populations as functions of time to produce the spectra associated with each mathematical component. 

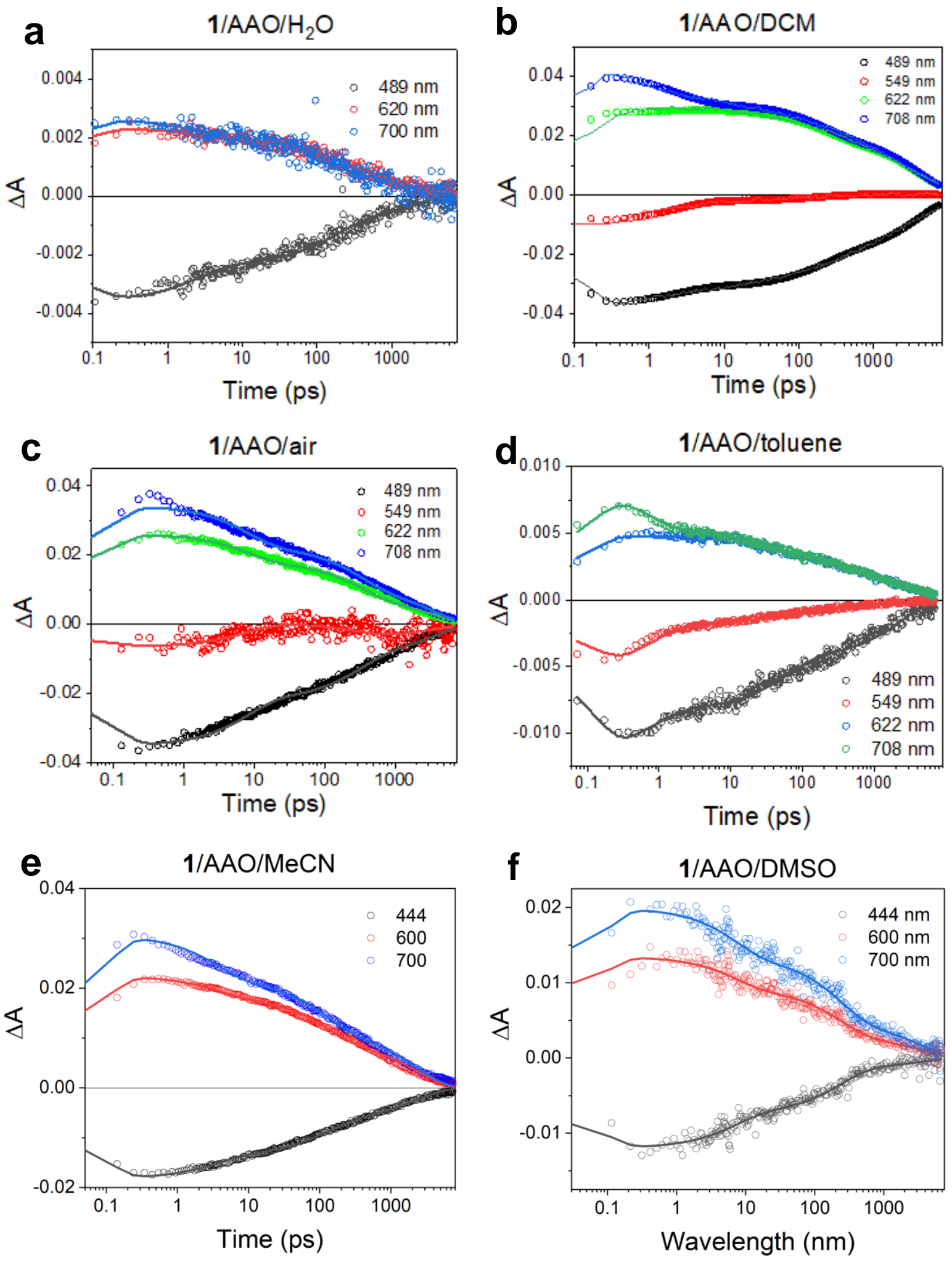

Figure S6. Kinetic fits for 1/AAO. $\left(\mathrm{DCM}=\mathrm{CH}_{2} \mathrm{Cl}_{2} ; \mathrm{MeCN}=\right.$ acetonitrile; DMSO = dimethylsulfoxide $)$ 

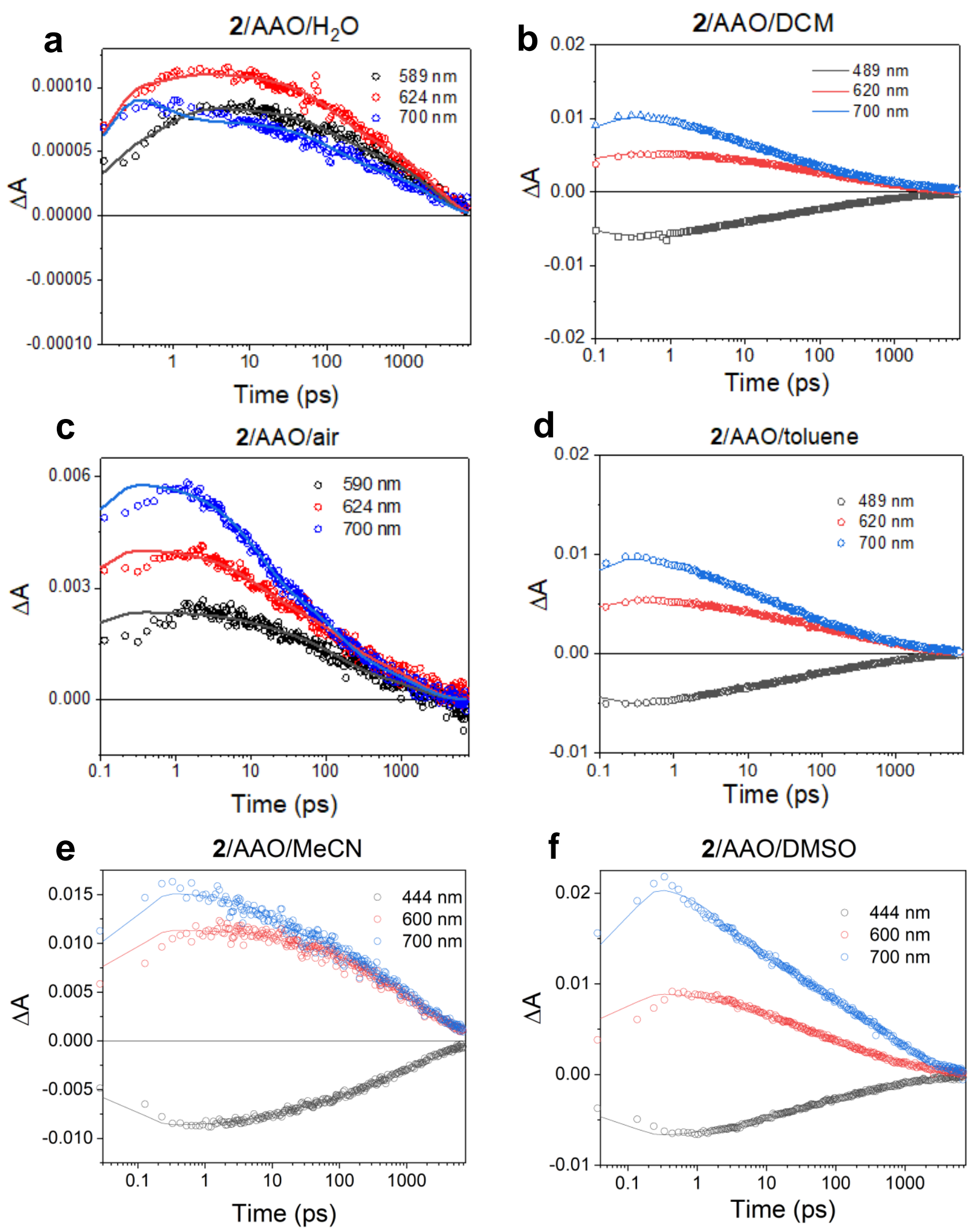

Figure S7. Kinetic fits for 2/AAO. (DCM $=\mathrm{CH}_{2} \mathrm{Cl}_{2} ; \mathrm{MeCN}=$ acetonitrile; DMSO = dimethylsulfoxide) 


\section{References}

1. Szpakolski, K.; Latham, K.; Rix, C.; Rani, R. A.; Kalantar-zadeh, K., Silane: A new linker for chromophores in dye-sensitised solar cells. Polyhedron 2013, 52, 719-732. 